\title{
Shape Reconstruction of Partially Overlapping Objects in SEM Images: Application to Silver Halide Microcrystals
}

\author{
Volodymyr V. Kindratenko, Boris A. Treiger and Pierre J.M. Van Espen \\ Micro- and Trace Analysis Centre (MiTAC), Department of Chemistry, \\ University of Antwerp (UIA), 2610 Antwerp, Belgium
}

(Received February 06; accepted February 25, 1997)

PACS.07.05.Pj - Image processing

PACS.42.70.Gi - Light-sensitive materials

PACS.61.16.Bg - Transmission, reflection and scanning electron microscopy (including EBIC)

\begin{abstract}
A method for shape reconstruction and extraction from objects that have a certain regularity but are observed in a scanning electron microscopy image with some degree of overlap is presented. The proposed algorithm first calculates the curvature at each contour point of the object in the digitized binary image in order to detect the vertexes. Reconstruction of the shape of overlapping objects is then based on geometrical considerations using the information from the vertex co-ordinates. The procedure is independent of the size and orientation of the objects. The method is applied to the shape reconstruction of partially overlapping tabular silver halide microcrystals.
\end{abstract}

\section{Introduction}

Scanning electron microscopy (SEM) is often used to study the shape and size of microscopic objects. E.g. this technique is applied to investigate atmospheric particles and industrial particulate materials such as ceramic powders and microcrystals. In combination with X-ray analysis (SEM-EDX) also the chemical composition of these microparticles can be obtained [1]. This type of studies involves the analysis of a large number of objects (typical between a few hundred to a few thousand) in order to obtain statistical valid results for the size, shape (and composition) distribution, so that automation of the procedure is often required.

In order to analyse the particle with SEM they need to be dispersed on a suitable conducting and flat substrate, e.g. by filtration of a suspension on a Nuclepore filter or by evaporating a drop of the suspension on a carbon stub. With carefully controlled sample preparation, agglomeration and overlap between the microparticles can be avoided to some extend but never excluded. Overlap occurs more frequently and is especially problematic with flat shaped objects such as tabular silverhalide microcrystals that have a diameter to thickness ration between 5 and 10 . One possibility is to disregard all cases of overlapping particles at the expense of an increased measurement time as one has to continue the measurement until the required number of non-overlapping objects has been identified and analysed.

In this article we propose an algorithm that is able to reconstruct the shape of the overlapping object based on geometrical considerations. The article emphasizes on the shape reconstruction 


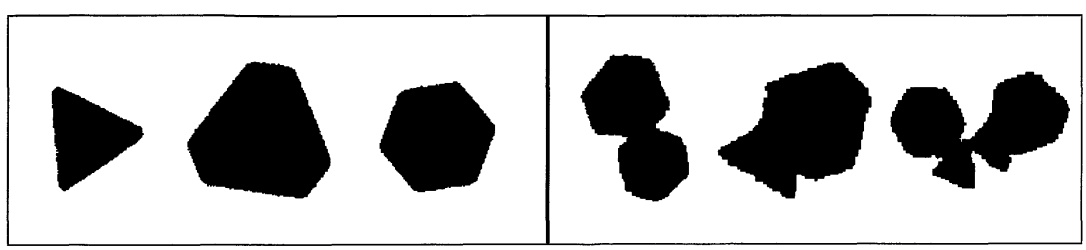

a)

b)

Fig. 1. - a) SEM backscattered electron images of individual triangular, truncated triangular and hexagonal silver halide microcrystals. b) The same microcrystals observed as partially overlapping objects in real samples.

of silver halide microcrystals but can be extended to other objects with a more or less regular shape.

The tabular silverhalide crystals are used in light sensitive photographic emulsions. They are produced in a reactor vessel by the precipitation reaction of $\mathrm{Ag}^{+}$with $\mathrm{Cl}^{-}, \mathrm{Br}^{-}$or I- ions. Their shape, size and composition is determined by the growth (precipitation) conditions and in turn determine the properties of the photographic material [2]. Knowledge about the size and shape distribution of these microcrystals is important for the optimization of the precipitation process and for the study of the photographic properties of light sensitive film. Under certain precipitation conditions a limited number of shapes are produced: regular triangles, regular hexagons and truncated triangles. Figure 1a shows binarized SEM backscattered electron images of some typical crystals. For the shape characterization and classification of these silver halide crystals methods have been proposed in the literature [3,4]. However these methods cannot be applied when one or more microcrystals are partially overlapping (Fig. 1b) as is often the case because of the limited control that one can exercise during the sample preparation process.

\section{Theory}

In the following we assume that the fact that two or more objects are overlapping each other has been established on the basis of a binary SEM image, e.g. using an approach described in [5]. The algorithm to extract the shape of the individual entities from such an ensemble (a collection of more or less overlapping objects) involves 4 steps. First an ordered 8-connected list of contour coordinates of the ensemble is extracted. In the second step this list is analysed and the vertexes are detected. Having found $N$ vertexes the ensemble is approximated with an $N$-side polygon. In the last two steps hexagonal and triangular (truncated and regular) shapes are reconstructed on the basis of the geometrical information obtained from the vertex positions.

\section{Contour Extraction}

The border following algorithm [6] is used for finding and storing the connected list of contour coordinates of the ensemble of overlapping objects. To illustrate this, assume that point $\mathrm{L}$ belongs to the ensemble and the point $\mathrm{R}$ not as shown in Figure 2a. This pair of the points defines one of the "cracks" on the border as called in [6]. We consider two other points $\mathrm{L}_{1}$ and $R_{1}$ which are 4 -adjacent to $L$ and $R$ respectively. Then the following rules define the next 

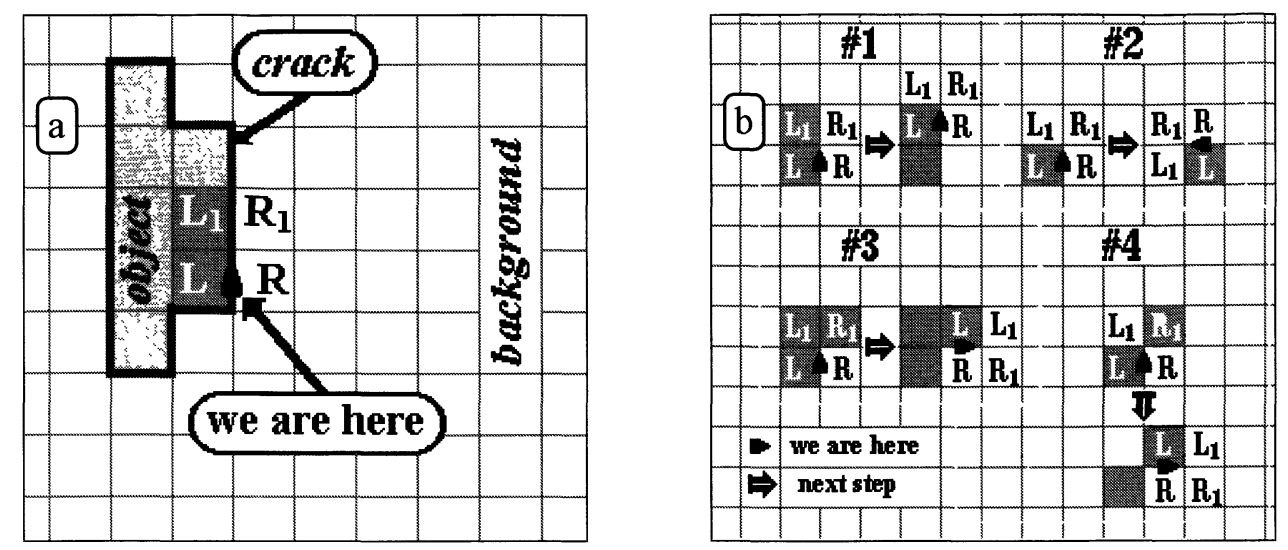

Fig. 2. - a) Definition of a crack and some important points. b) Illustrations of the rules of the border following algorithm.

crack on the border (see Fig. 2b):

$$
\begin{array}{ll}
\mathrm{L}_{\text {new }}=\mathrm{L}_{1}, \mathrm{R}_{\text {new }}=\mathrm{R}_{1} & \text { if } \mathrm{L}_{1} \text { belongs to the ensemble and } \mathrm{R}_{1} \text { not; } \\
\mathrm{L}_{\text {new }}=\mathrm{L}, \mathrm{R}_{\text {new }}=\mathrm{L}_{1} & \text { if } \mathrm{L}_{1} \text { and } \mathrm{R}_{1} \text { do not belong to the ensemble; } \\
\mathrm{L}_{\text {new }}=\mathrm{R}_{1}, \mathrm{R}_{\text {new }}=\mathrm{R} & \text { if } \mathrm{L}_{1} \text { and } \mathrm{R}_{1} \text { belong to the ensemble; } \\
\mathrm{L}_{\text {new }}=\mathrm{R}_{1}, \mathrm{R}_{\text {new }}=\mathrm{R} & \text { if } \mathrm{R}_{1} \text { belongs to the ensemble and } \mathrm{L}_{1} \text { not. }
\end{array}
$$

The algorithm terminates when we come back to the initial pair of points.

\section{Detection of Vertexes}

Vertex detection is based on the idea that if there is a vertex in a certain point of a contour then the change in tangent direction at that point will be essential. Thus in order to find vertexes, the tangent direction at each point of the contour is calculated and its change monitored.

To estimate the tangent direction, the "median filtered differencing technique" [7] is applied. Let $\left\{\mathrm{p}_{i}\right\}$ be an 8-connected list of contour coordinates, then at each point $\mathrm{p}_{i}$ let us define a set of $2 M$ difference vectors (Fig. 3a) $\mathbf{d}(i, i+j), j=-M, \ldots,-1,1, \ldots, M$, such that

$$
\mathbf{d}(i, i+j)=\left\{\begin{array}{ll}
\mathbf{d}\left(p_{i+j}, p_{i}\right), & j=-1, \ldots,-M \\
\mathbf{d}\left(p_{i}, p_{i+j}\right), & j=1, \ldots, M
\end{array} .\right.
$$

These difference vectors can be represented in polar coordinates and sorted according to their polar angle $\theta$. If $\theta_{k}$ be the angle of the $k$-th vector in the sorted sequence of $2 M$ vectors, the direction of tangent is estimated as a median of the sequence:

$$
\text { tangent direction }=\frac{1}{2}\left(\theta_{M}+\theta_{M+1}\right) .
$$

The algorithm effectively selects the median slope of the tangent direction as a function of arc length. The same idea was used for curvature estimation at each point of the contour (Fig. $3 \mathrm{~b}$ ).

It is assumed that point $\mathrm{P}$ of the contour is a vertex if the tangent direction at that point is larger than a certain limit value $\Delta \Theta$. It might occur that a few consecutive points have a tangent direction larger than $\Delta \Theta$. In that case the middle point in the sequence (the point with the highest tangent direction) is considered to be the vertex point. 

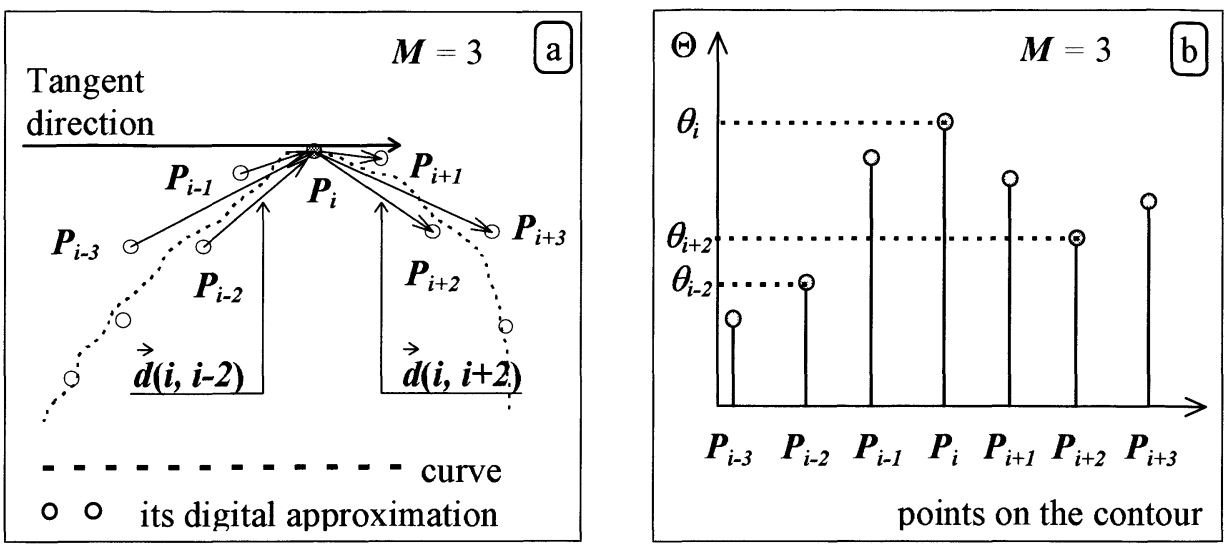

Fig. 3. - Median filtered differencing method. a) Sequence of points around $\mathrm{P}_{i}$ and the $2 M$ difference vectors, the tangent direction is defined as the median of corresponding angles of $\mathbf{d}(i, i+j)$. b) Curvature estimation, the curvature is the median of differences $\theta_{i}-\theta_{i+j}$.

\section{Extraction of Hexagonal Shaped Objects}

Both hexagonal and truncated triangular objects (silver halide microcrystals) have all vertexes equal to $120^{\circ}$. A regular hexagon has equal sides whereas a truncated triangle has sides of two different lengths. These properties and the parallelity of the opposite sides can be used to reconstruct partially overlapping objects of this category.

The angles between two vertexes of an $N$-sided polygon are determined with the cosine theorem. If an angle is between $\alpha_{1}$ and $\alpha_{\mathbf{u}}$ we mark the corresponding vertexes as belonging to a hexagonal object. The ordered sequences of vertexes, marked as belonging to an hexagonal object, are extracted and analysed separately in order to reconstruct the entire shape of the hexagon, if possible. If the vertex detection and marking procedures were correct one can expect to obtain sequences having $6,5,4,3,2$ or 1 vertexes. When the sequence has only 1 vertex we are not able to reconstruct the shape of corresponding hexagon. In case of 2 vertexes we can reconstruct only the shape of a regular hexagon but without faith in the true shape. Having 3, 4, 5 or 6 vertexes we can reconstruct the shape of regular hexagons and truncated triangular objects.

With 6 vertexes, the shape of the hexagon can be reconstructed simply by sequentially connecting of all of them (see Fig. 4a).

Having 5 vertexes, the shape of an hexagonal microcrystal can be reconstructed by adding the 6 th vertex using geometrical properties of the hexagon (see Fig. 4b). Let $\mathrm{P}_{1}, \mathrm{P}_{2}, \mathrm{P}_{3}$, $\mathrm{P}_{4}, \mathrm{P}_{5}$ be an ordered sequence of vertexes of an hexagon and $\mathrm{P}_{0}$ and $\mathrm{P}_{6}$ vertexes before $\mathrm{P}_{1}$ and after $\mathrm{P}_{5}$ respectively not belong to the hexagon (see Fig. $4 \mathrm{~b}$ ). The 6 th vertex $\mathrm{P}_{6}^{\prime}$ of the hexagon is at the interception of the lines $\mathrm{P}_{1} \mathrm{P}_{0}$ and $\mathrm{P}_{5} \mathrm{P}_{6}$. The coordinates of this point can be found as the solution of a system of the equations

$$
\left\{\begin{aligned}
\frac{y-y_{1}}{y_{0}-y_{1}} & =\frac{x-x_{1}}{x_{0}-x_{1}} \\
\frac{y-y_{5}}{y_{6}-y_{5}} & =\frac{x-x_{5}}{x_{6}-x_{5}}
\end{aligned}\right.
$$



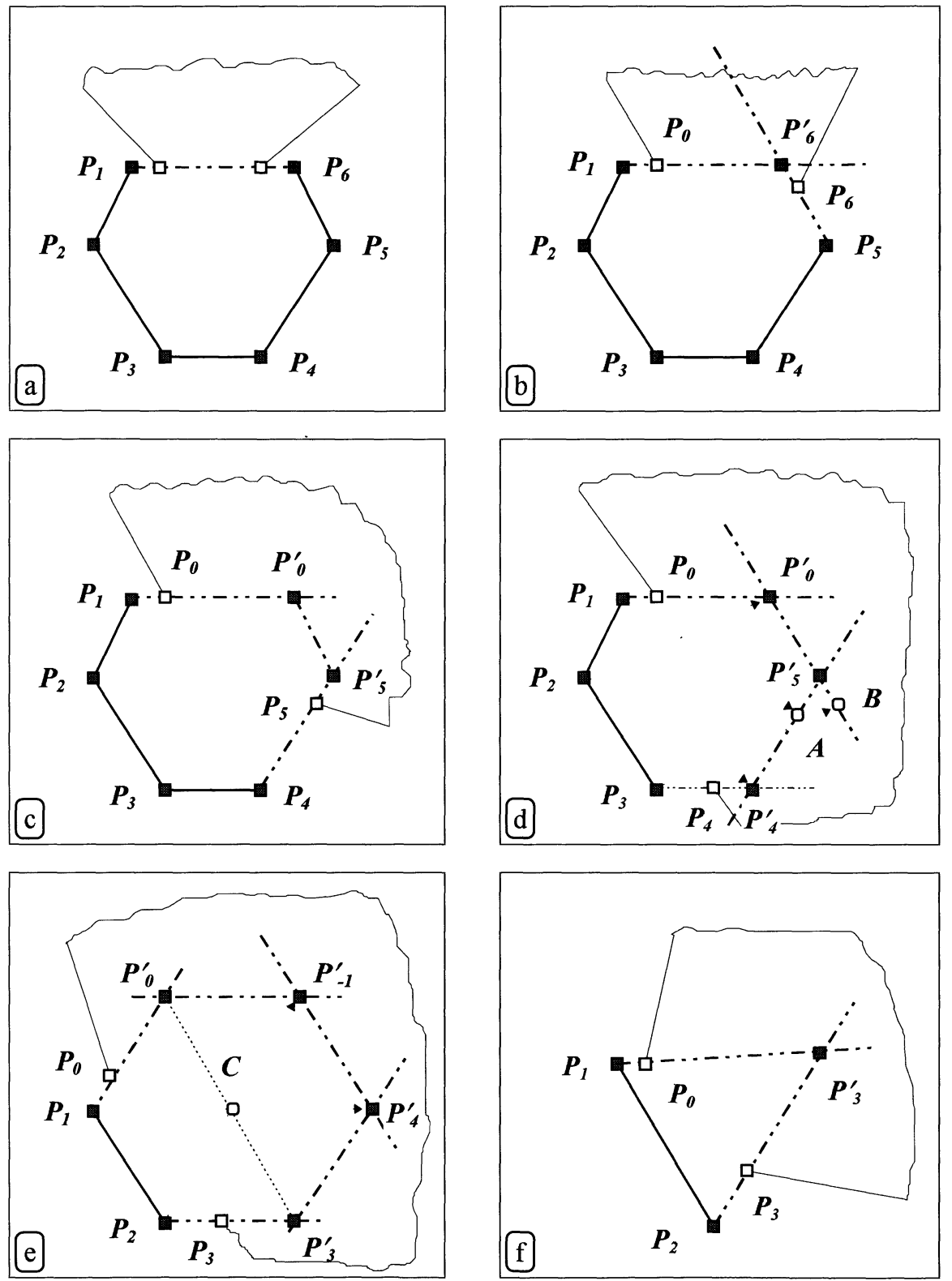

Fig. 4. - Illustration of the algorithms used for reconstruction of the shape of partially overlapping hexagonal and triangular objects.

representing the lines $\mathrm{P}_{1} \mathrm{P}_{0}$ and $\mathrm{P}_{5} \mathrm{P}_{6}$ passing through the points $\mathrm{P}_{0}\left(x_{0}, y_{0}\right), \mathrm{P}_{1}\left(x_{1}, y_{1}\right)$ and $\mathrm{P}_{6}\left(x_{6}, y_{6}\right), \mathrm{P}_{5}\left(x_{5}, y_{5}\right)$ respectively. Finally, having 6 vertexes the hexagon can be reconstructed as described above.

If only 4 vertexes of an hexagon are available the following procedure can be used for the reconstruction (see Fig. 4c). Let $\mathrm{P}_{1}, \mathrm{P}_{2}, \mathrm{P}_{3}, \mathrm{P}_{4}$, be an ordered sequence of points representing the vertexes of an hexagon and $\mathrm{P}_{0}$ and $\mathrm{P}_{5}$ vertexes before and after the given sequence 

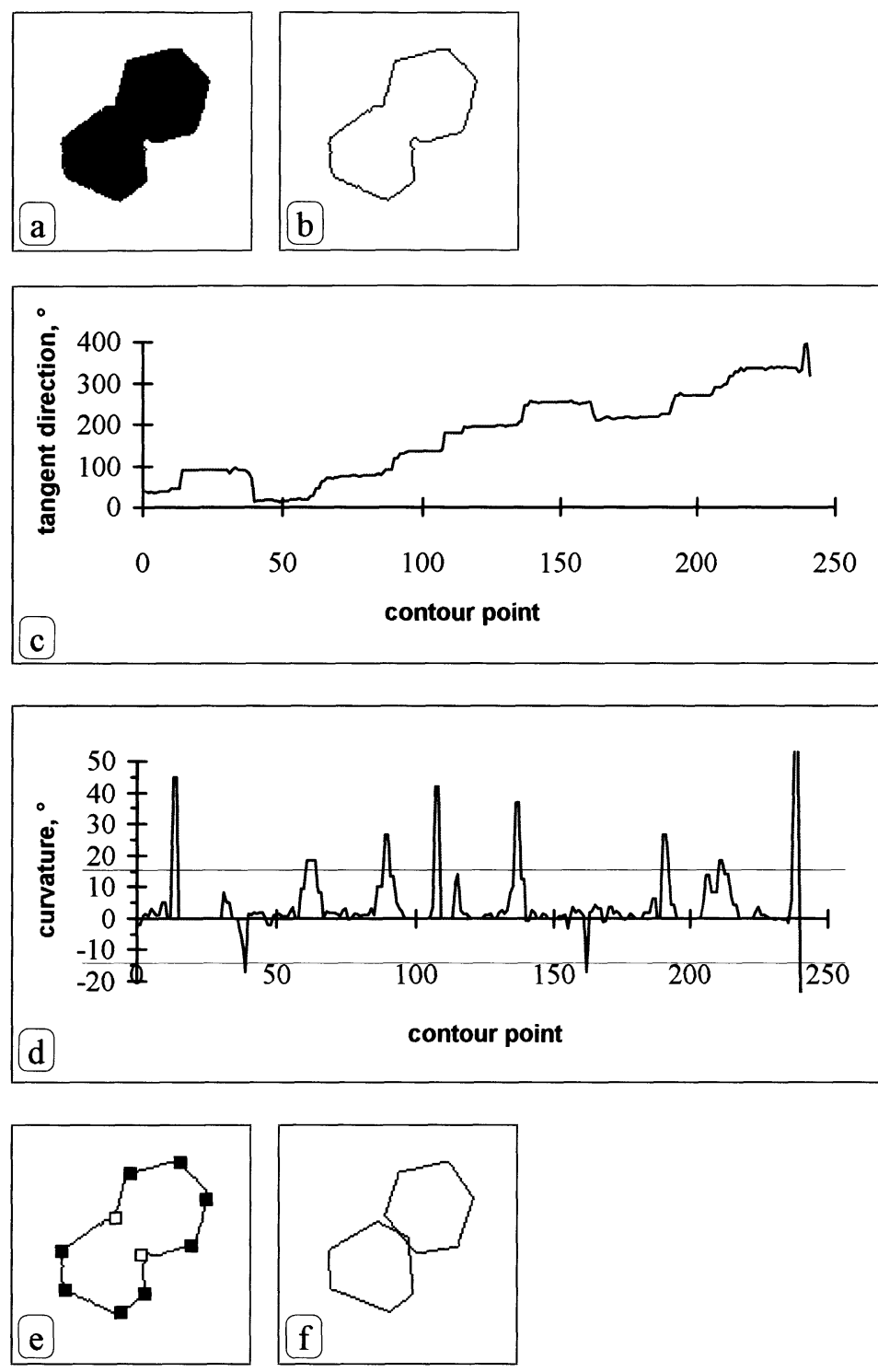

Fig. 5. - Illustration of the entire reconstruction procedure: a) binary image; b) extracted contour; c) estimated tangent direction at each point of the contour $(M=15)$; d) estimated curvature at each point of the contour $(M=2)$; e) vertexes belonging to hexagonal microcrystals are marked in black $\left(\Delta \Theta=15^{\circ}, \alpha_{1}=105^{\circ}, \alpha_{\mathrm{u}}=135^{\circ}\right) ;$ f $)$ contours of reconstructed microcrystals.

respectively. The 5th vertex of the hexagon $\mathrm{P}_{5}^{\prime}$ to be found belongs to the line $\mathrm{P}_{4} \mathrm{P}_{5}$ and the length of $\mathrm{P}_{4} \mathrm{P}_{5}^{\prime}$ is equal to the length of the side $\mathrm{P}_{2} \mathrm{P}_{3}$. So, its coordinates $x_{5}^{\prime}$ and $y_{5}^{\prime}$ can be calculated using the coordinates of the points $\mathrm{P}_{4}$ and $\mathrm{P}_{5}$ :

$$
x_{5}^{\prime}=x_{4}+l \frac{x_{5}-x_{4}}{d} \text { and } y_{5}^{\prime}=y_{4}+l \frac{y_{5}-y_{4}}{d}
$$

where $l$ is the length of $\mathrm{P}_{2} \mathrm{P}_{3}$ and $d$ is the length of $\mathrm{P}_{4} \mathrm{P}_{5}$. The 6 th vertex of the hexagon 


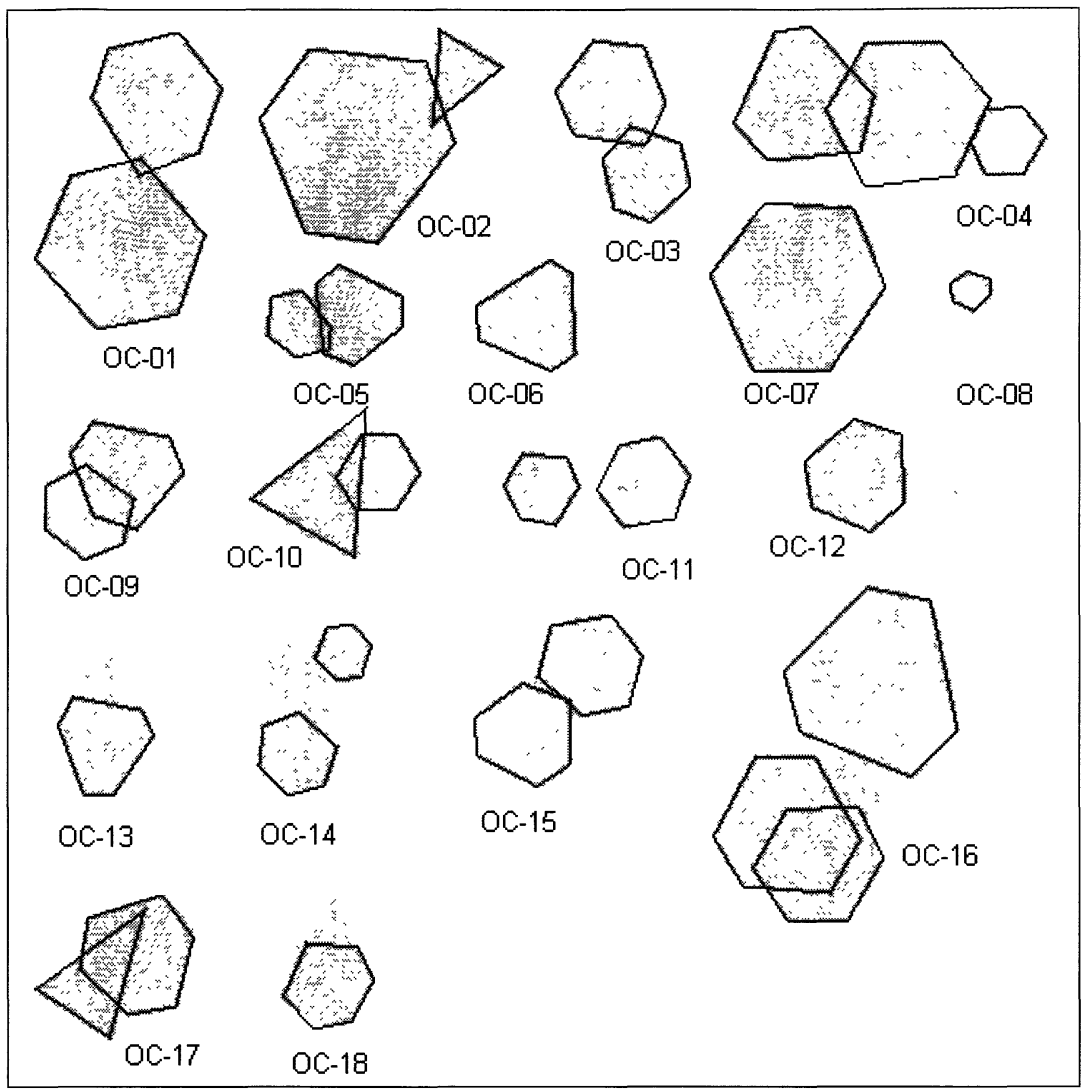

Fig. 6. - Results of the reconstruction of 18 ensembles of partially overlapping microcrystals.

$\mathrm{P}_{0}^{\prime}$ belongs to the line $\mathrm{P}_{1} \mathrm{P}_{0}$ and the length of $\mathrm{P}_{1} \mathrm{P}_{0}^{\prime}$ is also equal to the length of $\mathrm{P}_{2} \mathrm{P}_{3}$. Its coordinates can be found in a similar way using coordinates of the points $\mathrm{P}_{1}$ and $\mathrm{P}_{0}$.

Having only 3 vertexes of the hexagon, the remaining 3 can be found in the following way (see Fig. 4d). Let $\mathrm{P}_{1}, \mathrm{P}_{2}, \mathrm{P}_{3}$ be an ordered sequence of points representing the vertexes of an hexagon and $\mathrm{P}_{0}$ and $\mathrm{P}_{4}$ vertexes before and after this sequence. Vertexes of the hexagon $\mathrm{P}_{0}^{\prime}$ and $\mathrm{P}_{4}^{\prime}$ can be found in a similar way as described for 4 vertexes. The 6 th vertex $\mathrm{P}_{5}^{\prime}$ can be found as the interception of two lines parallel to lines $\mathrm{P}_{1} \mathrm{P}_{2}$ and $\mathrm{P}_{2} \mathrm{P}_{3}$ which cross the points $\mathrm{P}_{4}^{\prime}$ and $\mathrm{P}_{0}^{\prime}$, respectively. This interception can be found in a similar way as described for the case of 5 vertexes: the line passes the point $\mathrm{P}_{4}^{\prime}$ and is parallel to the $\mathrm{P}_{1} \mathrm{P}_{2}$ and also passes the point A with coordinates $\left(x_{1}+\left(x_{4}^{\prime}-x_{2}\right), y_{1}+\left(y_{4}^{\prime}-y_{2}\right)\right)$. Similarly, the line is passing the point $\mathrm{P}_{0}^{\prime}$ and is parallel to the side $\mathrm{P}_{2} \mathrm{P}_{3}$, also passes the point $\mathrm{B}$ with coordinates $\left(x_{3}+\left(x_{0}^{\prime}-x_{2}\right)\right.$, $\left.y_{3}+\left(y_{0}^{\prime}-y_{2}\right)\right)$.

In case only 2 vertexes of a hexagonal microcrystal were found we can only reconstruct a regular hexagonal microcrystal having all sides equal (see Fig. 4e). Let $\mathrm{P}_{1}$ and $\mathrm{P}_{2}$ be an ordered sequence of points representing the vertexes of a hexagon, $\mathrm{P}_{0}$ and $\mathrm{P}_{3}$ be one vertex before the given sequence and one vertex after the given sequence, as usually. Reconstruction of the regular hexagon is as follows. Two vertexes $\mathrm{P}_{0}^{\prime}$ and $\mathrm{P}_{3}^{\prime}$ of the hexagon can be found in the way similar to the case when 4 vertexes are available. The remaining 2 vertexes of the hexagon $\mathrm{P}_{-1}^{\prime}$ and $\mathrm{P}_{5}^{\prime}$ are defined using the property of central symmetry of a regular hexagon. 
The centre of symmetry $\mathrm{C}$ of the hexagon has coordinates $\left(\left(x_{0}^{\prime}+x_{3}^{\prime}\right) / 2,\left(y_{0}^{\prime}+y_{3}^{\prime}\right) / 2\right)$. The point $\mathrm{P}_{-1}^{\prime}$ belongs to the line $\mathrm{P}_{2} \mathrm{C}$ and the distance from the point $\mathrm{C}$ to the point $\mathrm{P}_{-1}^{\prime}$ is equal to the distance from the point $\mathrm{P}_{2}$ to the point $\mathrm{C}$. Thus coordinates of the point $\mathrm{P}_{-1}^{\prime}$ can be found using equations similar to that used in the case when only 4 vertexes are available. The last vertex $\mathrm{P}_{5}^{\prime}$ is defined in a similar way. Of course, in the many cases this reconstruction will be wrong and it will not always be possible to check the correctness of the reconstruction even by visual inspection.

\section{Extraction of Triangular Shaped Objects}

Partially overlapping triangular microcrystals can also be extracted based on their geometrical properties. This type of silver halide microcrystals are regular triangles with equal sides and vertexes of $\sim 60^{\circ}$.

After determining the numerical values of each angle of the $N$-sided polygonal, we mark the corresponding vertex as belonging to a triangular object if the angle is between $\beta_{1}$ and $\beta_{\mathrm{u}}$. Next, all continuous ordered sequences of vertexes previously selected as belonging to triangular objects are extracted and analysed separately in order to reconstruct the shapes. If the vertex detection and marking procedure is done correctly one expects to obtain sequences of vertexes having 3,2 or 1 points.

When a sequence has only 1 vertex we are obviously not able to reconstruct the corresponding triangular object correctly. If a sequence contains 3 points the shape of the object can be reconstructed simply by sequentially connection its vertexes.

When only two vertexes are available the 3 rd vertex of the triangular object can be determined as follows. Let $\mathrm{P}_{1}$ and $\mathrm{P}_{2}$ represent two real vertexes of a triangular object and $\mathrm{P}_{0}$ and $\mathrm{P}_{3}$ two vertexes which were detected in the overlapping ensemble: $\mathrm{P}_{0}$ is located before vertex $\mathrm{P}_{1}, \mathrm{P}_{3}$ located after vertex $\mathrm{P}_{2}$ (see Fig. $4 \mathrm{f}$ ). The coordinates of the 3 rd vertex $\mathrm{P}_{3}^{\prime}$, belonging to the triangular object, are found as the coordinates of the point of intersection of the lines $\mathrm{P}_{1} \mathrm{P}_{0}$ and $\mathrm{P}_{2} \mathrm{P}_{3}$ in the way similar to the one described above for the case of an hexagonal object having 5 vertexes.

\section{Results and Discussion}

Figure 5 illustrates the main steps of the reconstruction procedure. After the contour of the ensemble of overlapping objects is extracted (Fig. 5b) the tangent direction at every point of the contour is determined (Fig. 5c) and the curvature calculated (Fig. 5d). The tangent direction is estimated using $M=15$ and for the curvature estimation $M=2$ is used. A large value of $M$ in the case of tangent direction determination results in a smoothed estimate whereas a small value of $M$ in the case of the curvature calculation guarantees that all essential tangent direction changes are detected. There are many local extremes in the curvature plot as one can observe in Figure 5d. Most of them are caused by the digital nature of the contour and local imperfections of the crystals (noise). Only a few correspond to the real vertexes. Thus the value of $\Delta \Theta$ should be large enough to separate vertexes on the contour from noise. In all our cases $\Delta \Theta=15$ gives good results. In Figure $5 \mathrm{e}$ the vertexes recognized as belonging to hexagonal microcrystals are marked in black. The limiting angles $\alpha_{1}=105^{\circ}$ and $\alpha_{\mathrm{u}}=135^{\circ}$ were determined experimentally. With such limits we are able to eliminate vertexes due to noise and vertexes which do not belong to hexagonal objects. Finally, Figure $5 \mathrm{f}$ shows the reconstructed contour of the hexagonal and the truncated triangular microcrystals.

Eighteen ensembles of partially overlapping microcrystals are shown in Figure 6. They were analysed with the parameters listed above. The limiting angles for the detection of triangular 
objects were $\beta_{1}=40^{\circ}, \beta_{\mathbf{u}}=80^{\circ}$. In most cases our procedure is able to reconstruct the shape of partially overlapping microcrystals. For ensemble OC-04 the reconstructed shape of the hexagon is too large and two reconstructed hexagons of OC-14 are too small. In both cases the reconstruction was done having only 2 vertexes of the hexagons. Clearly a reconstruction based on only two vertexes is often subject to large errors.

\section{Conclusion}

The method described is able to reconstruct the shape of partially overlapping regular objects. The method is based on the analysis of geometrical information obtained from the position of the vertexes of an ensemble of overlapping objects and is independent of the orientation, location and size. The experimental results on silver halide microcrystals demonstrate that in most cases the shapes of the individual objects can be satisfactory reconstructed. The correctness of the reconstruction depends on the number of available vertexes.

\section{References}

[1] Van Espen P., Quantitative microbeam analysis of particles, Mikrochim. Acta 114/115 (1994) 129-142.

[2] Geuens I., Development of Micro and Surface Analysis Methods for the Quantitative Characterzation of Silver Halide Microcrystals, PhD Dissertation, University of Antwerp (1993).

[3] Dougherty E., Mc Cabe J., Thurston E. and Laskin J., Automated Classification of Silver Halide Crystals, Imaging Sci. Technol. 47-50 (1990).

[4] Nys B., The Development of Image Processing and Analysis Techniques for Applications in Chemistry and Biomedicine, PhD Dissertation, University of Antwerp (1991).

[5] Kindratenko V.V., Van Espen P.J.M., Treiger B.A. and Van Grieken R.E., Characterisation of the shape of microparticles via fractal and Fourier analyses of their SEM images, Microchim. Acta [Suppl.] 13 (1996) 355-361.

[6] Rosenfeld A. and Kak A.C., Digital Picture Processing, vol. 2 (Academic Press, London, 1982).

[7] Matas J., Shao Z. and Kittler J. Estimation of Curvature and Tangent Direction by Median Filtered Differencing, Lect. Notes Comput. Sci. 974 (1995) 83-88. 